\title{
IMPACTO DE LOS BIOMARCADORES EN EL GLAUCOMA PRIMARIO DE ÁNGULO ABIERTO
}

\section{IMPACT OF BIOMARKERS IN PRIMARY OPEN-ANGLE GLAUCOMA}

\author{
ZANÓN-MORENO VC ${ }^{1}$, PINAZO-DURÁN MD²
}

\begin{abstract}
¿Qué es un marcador biológico? Según el diccionario de la Real Academia de la Lengua es todo átomo o sustancia detectable con cierta facilidad, que permite identificar procesos físicos, químicos o biológicos. En biomedicina nos referimos a ellos como las moléculas que podemos determinar en los tejidos y fluidos corporales mediante técnicas de laboratorio, y que según sus características, función y disponibilidad, nos facilitan el diagnóstico y el pronóstico de los procesos nosológicos. La principal utilidad de un biomarcador es como indicador de un estado concreto, y como ayuda indispensable para el reconocimiento de los sujetos más vulnerables en una población determinada. Si tenemos en cuenta que existen aproximadamente unas 600 enfermedades neurológicas, y que solamente unos pocos biomarcadores están disponibles para ellas, lógicamente deberemos favorecer este campo de la investigación. Algunos de éstos se han relacionado bien con procesos normales y patológicos, bien como respuesta farmacológica frente a determinadas terapias. Se han descrito marcadores ligados a procesos de inflamación, isquemia-reperfusión, cáncer y neurodegeneración, entre otros (1).

El glaucoma es una de las principales causas de ceguera mundial. Entre los tipos de glaucoma, el más frecuente es el primario de ángulo abierto (GPAA), constituyendo aproximadamente el 60\% de todos los casos. El comienzo es asintomático, detectándose en ocasiones por la hipertensión ocular (HTO), y en otras por la pérdida de visión, siendo esta última la manifestación del daño irreparable (2). Es por esto que el estudio de los signos y síntomas que informen acerca de los estadios iniciales y
\end{abstract}

de la progresión de la enfermedad glaucomatosa son primordiales para evitar la ceguera de los pacientes afectos. En los últimos años se han desarrollado estrategias para identificar nuevas moléculas implicadas en esta enfermedad que, junto a otras ya conocidas, pueden ayudar a establecer los mecanismos etiopatogénicos de la misma. Ante todo, hay que considerar que el glaucoma integra un grupo de procesos bien distintos, cuyo resultado final es la muerte de las células ganglionares de la retina que se refleja en la pérdida de las fibras del nervio óptico, que a su vez se manifiestan funcionalmente en la perdida de campo visual y excavación de la cabeza del nervio óptico. Los glaucomas en los que predomina la HTO se caracterizan por alteración de la malla trabecular, con resultado de la disminución de la facilidad de salida del humor acuoso. Los glaucomas con mecanismos independientes de la presión intraocular (PIO) están asociados a factores poco conocidos en el contexto de la enfermedad glaucomatosa, entre los que cabe destacar la isquemia, inflamación, predisposición genética, enfermedades cardiovasculares, procesos autoinmunes, hábitos tóxicos, etc. La HTO lesiona las estructuras del ángulo de la cámara anterior, provocando el depósito de pigmento y fibras elásticas, las calcificaciones y anomalías diversas en las células endoteliales de la pared interna del canal de Schlemm (3). En el transcurso de la enfermedad se produce selectivamente la afectación del tejido neural, con lesión y muerte por apoptosis de las células ganglionares de la retina y la pérdida de fibras ópticas.

$\mathrm{Si}$ tenemos en cuenta el conocimiento actual sobre el tema, ¿podemos afirmar que existen bio-

\footnotetext{
1 Doctor en Biología. Universidad de Valencia. Unidad Investigación Oftalmológica Santiago Grisolía. H. U. Dr. Peset. Valencia. E-mail: zanon_vicmor@gva.es

2 Doctor en Medicina y Cirugía. Especialista en Oftalmología. Unidad Investigación Oftalmológica Santiago Grisolía. H. U. Dr. Peset. Valencia. E mail: pinazo_mar@gva.es
} 
marcadores fiables para la enfermedad glaucomatosa? Lamentablemente, hasta este momento no hay moléculas que específicamente puedan relacionarse con el glaucoma, aunque la investigación actual se dirige hacia la búsqueda de marcadores que nos ayuden a: 1) distinguir entre los tipos de glaucoma, 2) estimar la progresión de la enfermedad, 3) identificar la población de mayor riesgo y 4) abordar el diseño de terapias específicas o, lo que es lo mismo, el desarrollo de tratamientos personalizados. Entre las líneas prioritarias en las que se centran estas investigaciones están el estrés oxidativo, la supervivencia y muerte celular y la expresión de genes. Vamos a revisarlos punto por punto.

Estrés Oxidativo.- Se ha relacionado etiopatogénicamente con las anomalías de la malla trabecular y con la apoptosis de las células ganglionares en el GPAA. Ya en 1996, Tamm y cols. (4) relacionaron estas lesiones con el estrés oxidativo, concluyendo que la sobre-expresión de la proteína alfa B-cristalina en cultivos celulares de la malla trabecular, podía relacionarse con la intensidad del daño glaucomatoso. Posteriormente, Izzotti y cols (5) demostraron que existía daño oxidativo sobre el ADN de las células de la malla trabecular en pacientes con GPAA, que se manifestaba por aumento significativo de la 8-hidroxiguanosina. Ferreira y cols (6) estudiaron la actividad enzimática antioxidante superóxido dismutasa y glutation peroxidasa y la capacidad antioxidante total en el humor acuoso de glaucomatosos, concluyendo que éstos pueden servir como marcadores, ya que el estrés oxidativo provoca la disminución global de la actividad antioxidante. Y en esta misma línea, otros trabajos han demostrado "in vitro" la presencia de metabolitos de la peroxidación lipídica, como el malonildialdehído (MDA) y el 4-hidroxinonenal (4HNE) en relación a la lesión glaucomatosa, observando el daño selectivo a las células ganglionares que puede contrarrestarse por los enzimas antioxidantes (7). Nuestro grupo de investigación ha demostrado la presencia de estrés oxidativo en el humor acuoso de pacientes programados para cirugía antiglaucomatosa. Entre los aspectos no elucidados, destaca el determinar si el estrés oxidativo es la causa principal de la enfermedad glaucomatosa o bien la consecuencia de la misma.

Supervivencia y Muerte Celular.- Diversos grupos de investigación, entre ellos los de E. Vecino, M.P. Villegas, M. Vidal, I. Vinuesa, J.M. Ramírez Sebastián y el nuestro, han estudiado los mecanis- mos de supervivencia y muerte celular en el glaucoma, con particular interés en la apoptosis y la neuroprotección. Existen evidencias que apoyan la inducción de apoptosis por el estrés oxidativo, y que este proceso es detectable mediante técnicas de laboratorio relativamente sencillas, pudiendo relacionarse con la progresión de la enfermedad glaucomatosa (8). Dentro de la complejidad de la cascada apoptótica destacan, como biomarcadores de apoptosis, las caspasas (particularmente la caspasa3) y la poli (ADP-ribosa) polimerasa (PARP-1). Se ha demostrado que el PARP 1 puede ser utilizado como marcador de apoptosis en el GPAA, aunque existe controversia en cuanto a la utilidad de la caspasa-3 (9). Otros estudios demuestran la utilidad de la proteína bcl-2 como marcadora de supervivencia celular, pues inhibe la muerte por apoptosis. En este sentido, debemos mencionar también al factor derivado del epitelio pigmentado (PEDF) y fibroblástico básico (bFGF) como otros importantes marcadores de supervivencia. Diversos trabajos describen su función neuroprotectora frente a estímulos que inducen muerte neuronal apoptótica en neuronas de la retina y se han propuesto como potencialmente útiles en el desarrollo, mantenimiento y función de las células retinianas. Continuando con este concepto, las células neurogliales cumplen funciones esenciales para el mantenimiento de las neuronas. Debido a sus relaciones con los vasos sanguíneos y axones ópticos, intervienen directamente en el equilibrio electrolítico y metabolismo de neurotransmisores (glutamato), producen laminina, fibronectina y tropoelastina, y también diversos factores de crecimiento (bFGF, TGF- b) o supervivencia neuronal (NGF) (10).

Expresión de genes.- Existen numerosos factores de riesgo para el glaucoma, como la edad, la raza, la miopía severa, la diabetes, etc. Otro de estos factores de riesgo es la historia familiar de glaucoma; de hecho, aproximadamente un $20 \%$ de los sujetos con glaucoma presentan antecedentes familiares (11), lo que nos indica que esta enfermedad presenta un componente genético importante. Un dato que apoya esta afirmación es que la prevalencia del GPAA aumenta de un 2,8\% en la población general hasta un $13,5 \%$ entre familiares de pacientes, así como el hecho de que muchos parámetros oculares sean de naturaleza hereditaria, como la presión intraocular, la resistencia al flujo ocular, la excavación papilar y las dimensiones oculares (profundidad de la cámara anterior y longitud axial del 
ojo) (12). Se han realizado numerosos estudios siguiendo esta línea de investigación y se ha demostrado la asociación de diversos genes con esta enfermedad, como el gen de la miocilina, el de la optineurina o el gen CYP1B1, lo que revela que el carácter hereditario del glaucoma primario de ángulo abierto no se encuentra en un solo gen, sino en varios. Además, quizá no baste con la presencia de los polimorfismos asociados al glaucoma, sino que sea la interacción genes-ambiente la que posibilite la aparición de la enfermedad.

En definitiva, son muchos los biomarcadores estudiados en relación al glaucoma primario de ángulo abierto. A pesar de ello, y de lo mucho que aún falta por dilucidar a este respecto, podemos afirmar que el estudio y la comprensión de las bases genéticas y moleculares del glaucoma primario de ángulo abierto es fundamental para alcanzar en un futuro nuevas terapias que nos permitan frenar la progresión de la enfermedad y evitar la ceguera glaucomatosa.

\section{BIBLIOGRAFÍA}

1. Schulte I, Tammen H, Selle H, Schulz-Knappe P. Peptides in body fluids and tissues as markers of disease. Expert Rev Mol Diagn 2005; 5: 145-157.

2. Distelhorst JS, Hughes GM. Open-angle glaucoma. Am Fam Physician 2003; 67: 1937-1944.

3. Alvarado JA, Alvarado RG, Yeh RF, Franse-Carman $L$, Marcellino GR, Brownstein MJ. A new insight into the cellular regulation of aqueous outflow: how trabecular meshwork endothelial cells drive a mechanism that regulates the permeability of Schlemm's canal endothelial cells. Br J Ophthalmol 2005; 89: 1500-1505.

4. Tamm ER, Rusell P, Johnson DH, Piatigorsky J. Human and monkey trabecular meshwork accumulate alpha??? crystallin in response to heat shock and oxidative stress. Invest Ophthalmol Vis Sci 1996; 37: 2402-2413.

5. Izzotti A, Saccà SC, Cartiglia $C$, De Flora S. Oxidative deoxyribonucleic acid damage in the eyes of glaucoma patients. Am J Med 2003; 114: 638-646.

6. Ferreira SM, Lerner SF, Brunzini R, Evelson PA, Llesuy SF. Oxidative stress markers in aqueous humor of glaucoma patients. Am J Ophthalmol 2004; 137: 62-69.

7. Malone PE, Hernandez MR. 4-Hydroxinonenal, a product of oxidative stress, leads to antioxidant response in optic nervehead astrocytes. Exp Eye Res 2007; 84: 444-454.

8. He Y, Leung $K W$, Zhang YH, Duan S, Zhong XF, Jiang RZ, et al. Mitochondrial complex I defect induces $R O S$ release and degeneration in trabecular meshwork cells of POAG patients: protection by antioxidants. Invest Ophthalmol Vis Sci 2008; 49: 1447-1458.

9. Li GY, Osborne NN. Oxidative-induced apoptosis to inmortalized ganglion cell lines is caspase independent but involves the alteration of poly (ADP-ribose) polymerase and apoptosis-inducing factor. Brain Res 2008; 1188: 35-43.

10. Ramírez JM, Salazar JJ. Implicación de la astroglía en el daño glaucomatoso. Arch Soc Esp Oftalmol 2008; 83: 339-342.

11. Ray K, Mukhopadhyay A, Acharya M. Recent advances in molecular genetics of glaucoma. Mol Cell Biochem 2003; 253: 223-231.

12. van Koolwijk LM, Despriet DD, van Duijn CM, Pardo Cortes LM, Vingerling JR, Aulchenko YS, et al. Genetic contributions to glaucoma: heritability of intraocular pressure, retinal nerve fiber layer thickness, and optic disc morphology. Invest Ophthalmol Vis Sci 2007; 48: 36693676. 